\title{
Modal parameter identification of all-GFRP composite cable-stayed footbridge in Denmark
}

\author{
Piotr Górski ${ }^{1, *}$, Beata Stankiewicz ${ }^{1}$ and Marcin Tatara ${ }^{1}$ \\ ${ }^{1}$ Opole University of Technology, Faculty of Civil Engineering and Architecture, Department \\ of Roads and Bridges, Katowicka 48, Opole 45-061, Poland
}

\begin{abstract}
The aim of this paper is to investigate of dynamic characteristics of cable-stayed Fiberline Bridge in Kolding, Denmark, made entirely of Glass Fiber Reinforced Polymer (GFRP) composite. During examination based on in situ free-decay measurements and using accelerometers under human jumping the primary five natural frequencies, corresponding mode shapes and damping ratios of the footbridge were identified. The Peak Picking (PP) and Frequency Domain Decomposition (FDD) approaches were applied to identify the natural frequencies and mode shapes. The corresponding damping ratios were extracted by a linear regression on the extremes of modal decays. The estimated damping ratios were compared with published data for selected footbridges made of various conventional materials. The obtained experimental results provide a relevant data regarding the dynamic response prediction or structural health monitoring of all-GFRP composite footbridges.
\end{abstract}

\section{Introduction}

Nowadays, Glass Fiber Reinforced Polymer (GFRP) composite material is one of the most innovative material being slowly introduced in a bridge structures. The decisive meaning has several key advantages of the material such as relatively high strength, low self-weight, ease of installation, corrosion resistance, electromagnetic transparency and ease of creation an innovative shapes of pultruded profiles as well as whole structure. Besides the advantages mentioned above, GFRP has also some limitations like a relatively low Young's modulus, low alkaline resistance and low long-term strength due to stress rupture [1-2]. For this reason, the examinations of mechanical properties and durability of GFRP material are still being performed, e.g. [3], in order to improve this technology as well as to increase the use of this material in civil engineering structures.

Currently, the GFRP profiles are willingly combined with some conventional materials, like steel or concrete, in a modern hybrid footbridges and are usually used as a bridge deck system. Some examples of such structures are given in the papers [4-5]. There are only few all-composite bridges reported in the literature. Among them the following footbridges can be mentioned: the cable-stayed Scripps Bridge in La Jolla, USA [6], Aberfeldy Bridge in Scotland, UK [7], or Fiberline Bridge in Kolding, Denmark (considered in this paper), as

\footnotetext{
*Corresponding author: p.gorski@po.opole.pl
} 
well as the Pontresina Bridge in Switzerland [8] with a truss structural system or the girders footbridge constructed in Tainan, Taiwan [9].

The knowledge of an actual value of modal characteristics of the structure is very useful for the procedure of calculation model verification, calculation model updating, dynamic response prediction, assessment of the technical state or structural health monitoring of the structures. It is very important issue, especially, in the case of all-GFRP footbridges for which the lack of specific design codes and insufficient number of studies in existing literature in this matter (e.g. [10-11]) can be observed. At a design stage, the natural frequencies and corresponding mode shapes are usually obtained from a solution of a free vibration response for an idealized calculation model. Unfortunately, the damping ratio, which is one of the most important and uncertain parameter significantly reducing the reliability of dynamic response prediction, could not be estimated basing on theoretical method. Thus, it is very difficult to predict damping parameters with a great certainty. The most appropriate way to investigate the actual dynamic characteristics of existing structures is to analyze their dynamic response recorded in a full scale. Nowadays, it is very advisable and valuable to conduct some studies in order to recognize the fundamental dynamic characteristics of all-GFRP footbridges.

The paper concerns with the investigation of dynamic characteristics of cable-stayed Fiberline Bridge in Kolding, Denmark, made entirely of GFRP composite material. During investigation based on in situ free-decay measurements, using accelerometers under human jumping, the primary five natural frequencies, corresponding mode shapes and damping ratios of the footbridge were identified. The Peak Picking (PP) and Frequency Domain Decomposition (FDD) approaches were applied to identify the natural frequencies and mode shapes. The corresponding damping ratios were extracted by a linear regression on the extremes of modal decays. The estimated damping ratios were compared with data published in the literature for selected footbridges made of various conventional materials. The obtained experimental results provide relevant data for the dynamic response prediction and structural health monitoring of all-GFRP composite footbridges.

\section{Description of the Fiberline Bridge in Kolding}

The cable-stayed Fiberline Bridge, located in Kolding city in Denmark, was constructed in 1997 using 12 different pultruded profiles all made of GFRP material. The footbridge is crossing an overhead main railway line near a salt water fjord. In spite of these disadvantageous conditions, the expected life time of the structure is minimum 100 years. The general view of the footbridge and its longitudinal section with the most important dimensions are shown in Fig. 1.

(a)

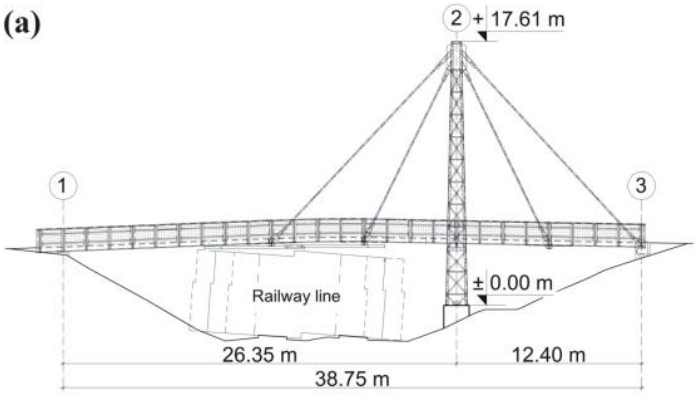

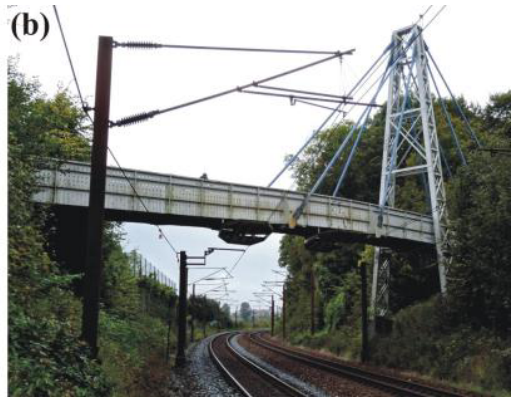

Fig. 1. Fiberline Bridge in Kolding: (a) longitudinal section and (b) general view.

The $40.3 \mathrm{~m}$ long and $3.21 \mathrm{~m}$ width footbridge comprises two continuous spans with lengths of 27.9 and $12.4 \mathrm{~m}$, supported by a single A-shaped $18.5 \mathrm{~m}$ tall pylon. Four pairs of 
cables with a square cross-section and a length of about 17.8, 17.6, 13.6 and $13.4 \mathrm{~m}$ are secured to the top of the pylon. The structural system of the deck comprises two I-shaped pultruded profiles with a height of $1.4 \mathrm{~m}$ used as main girders. Total weight of the bridge is just 12 tonnes. The additional information about the footbridge is given in the Fiberline Composites Website [12].

\section{Experimental set-up and measurement results}

In order to identify the modal parameter of the considered footbridge a free structural response for excitation under human jumping was measured using accelerometers. Since no significant lateral vibrations of the bridge were exhibited only the vertical acceleration component was measured. Due to the necessity of the natural modes determination, two accelerometers were used simultaneously in the field test. In total 28 measurement points were chosen directly on the upper edge of two girders, namely girders denoted as " $A$ " and "B", mainly within $3.1 \mathrm{~m}$ distance along the longitudinal axis of the girders. The measurement points are shown in Fig. 2. During the field measurement one reference accelerometer was fixed at the reference point, while the second rover accelerometer was moved step by step from a measurement points No. 1 to 27 . With every rearrangement of the rover accelerometer, i.e. at each measurement point, the series of two human jumps were performed at the deck center near the reference point and the free vibration responses of the footbridge were recorded during at least $25 \mathrm{~s}$ with the sampling rate of $200 \mathrm{~Hz}$. The reference point was chosen at a point where it was assumed that the most important modes have the most significant modal component. Fig. 3(a) depicts vertical accelerations of the footbridge girder " $\mathrm{A}$ " at the reference point and measurement point No. 23 surveyed due to two human jumps.

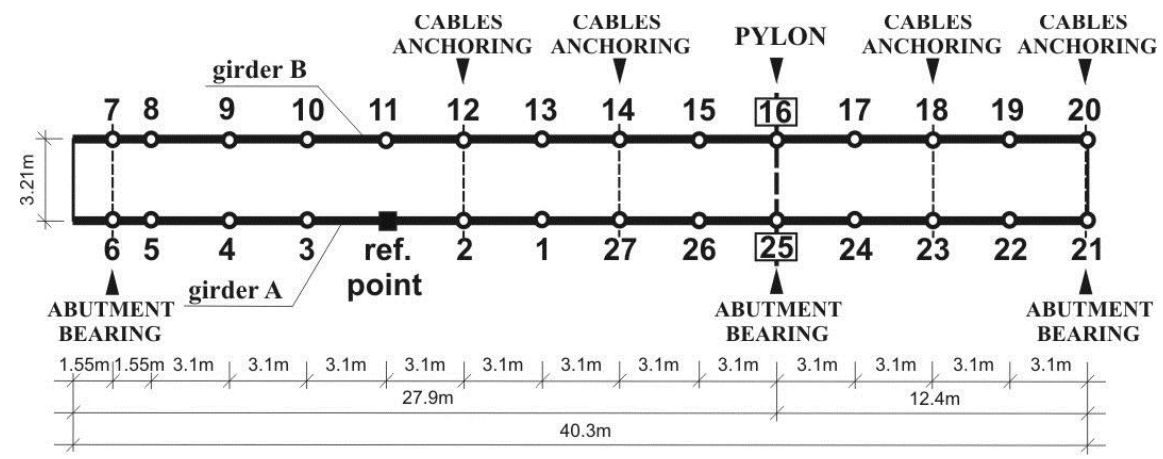

Fig. 2. Locations of the measurement points on the girders denoted as "A" and "B" of the footbridge.

(a)

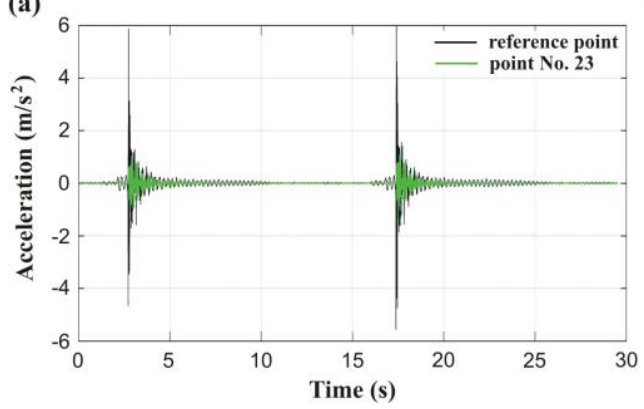

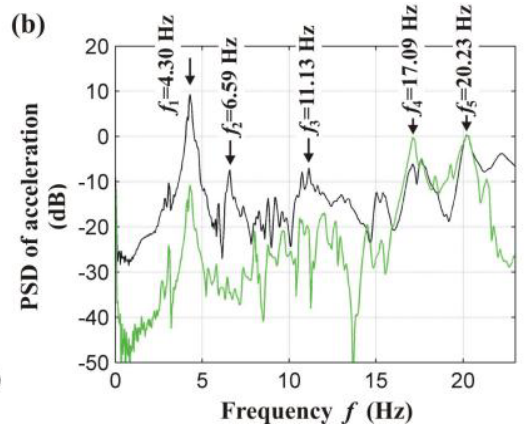

(b)

Fig. 3. (a) Recorded free-decay vertical vibrations of the footbridge girder "A" at the reference point and measurement point No. 23 excited by two human jumps and (b) their PSDs. 
In the field test two uniaxial high sensitivity accelerometers type PCB 3711E112G, based on the Micro-Electro-Mechanical System (MEMS) technology, were used. The measurement range of the sensors was $\pm 2 \mathrm{~g}$, the frequency range $0-400 \mathrm{~Hz}$, the broadband resolution $0.1 \mathrm{mg} \mathrm{rms}$ in 0.5 to $100 \mathrm{~Hz}$ bandwidth, and the spectral noise $15 \mu \mathrm{g} / \sqrt{\mathrm{Hz}}$ in 1 to $100 \mathrm{~Hz}$ bandwidth. Such sensitivity is adequate for studying behaviour of the footbridge.

All measured free-decays were analysed by the Fast Fourier Transform. The Power Spectral Density (PSD) functions were estimated for the total duration of the time interval of all free vibration responses. The PSDs of measured vertical accelerations of the footbridge girder " $A$ " at the reference point and measurement point No. 23 are presented in Fig. 3(b). These PSDs are dominated by five well-separated peaks with the values above 10 $\mathrm{dB}$, representing the lowest five natural frequencies, i.e. $f_{1}=4.30 \mathrm{~Hz}, f_{2}=6.59 \mathrm{~Hz}$, $f_{3}=11.13 \mathrm{~Hz}, f_{4}=17.09 \mathrm{~Hz}$, and $f_{5}=20.23 \mathrm{~Hz}$. In accordance with further analysis performed using the FDD method, presented in Section 4, it was found that these five frequencies correspond with specific natural mode shapes of the considered footbridge.

\section{Modal parameter estimation}

\subsection{Mode shapes identification using FDD technique}

The natural mode shapes of the footbridge were evaluated using the FDD method based on the free decays time series measured for all arrangement of two accelerometers at the reference point and measurement points denoted from No. 1 to 27 (see Fig. 2).

The FDD technique was introduced by Brincker in 2000 [13] as one of non-parametric frequency domain signal processing methods used in an output-only modal analysis. The method is appropriate to extract the natural frequencies and corresponding mode shapes using the Singular Value Decomposition (SVD) of the PSD matrices of a structural responses at each discrete frequency calculated with the assumption that the structural damping is light. Such decomposition is performed in order to identify a structure as a single degree of freedom (SDOF) system for each singular value corresponding to the resonant frequency of the structure and corresponding to the singular vector containing the information about the mode shape of the structure.

The first step of FDD is the estimation of a set of the PSD matrices of the structural response at each discrete frequency. All the matrices are Hermitian, i.e. the matrices are symmetric with complex conjugate elements around the diagonal. For example, in case of the considered field measurement of the footbridge response performed simultaneously in two measuring points the PSD matrix $\mathbf{G}_{x x}$ of the response at an $i$-th discrete frequency $f_{i}$ is expressed as follows

$$
\mathbf{G}_{x x}\left(\mathrm{i} f_{i}\right)=\left[\begin{array}{ll}
P S D_{11}\left(\mathrm{i} f_{i}\right) & C S D_{12}\left(\mathrm{i} f_{i}\right) \\
C S D_{21}\left(\mathrm{i} f_{i}\right) & P S D_{22}\left(\mathrm{i} f_{i}\right)
\end{array}\right]
$$

where $P S D(i f)$, as the elements on the diagonal, are the magnitudes of the PSD of the structural response at a chosen point of the structure, which for a real stochastic process $x(t)$ are real elements, $C S D(\mathrm{i} f)$, as the elements on the off-diagonal, are the cross PSD of the structural response at two various points of the structure and are the complex conjugate, Hermitian's PSDs, which fulfill equation $C S D_{q r}(\mathrm{i} f)=C S D^{*}{ }_{r q}(\mathrm{i} f)$, whereas $q \neq r$, the symbol * denotes a complex conjugate value, and $\mathrm{i}=\sqrt{-1}$ is the imaginary unit.

Since the FDD technique is well-known in the existing literature, e.g. [13], the further explanations are omitted. It should be noted that the final form of the PSD matrix of the structural response could be decomposed at each discrete frequency $f_{i}$ into a set of singular 
values and corresponding singular vectors using SVD according to the following expression [13]

$$
\mathbf{G}_{x x}\left(\mathrm{i} f_{i}\right)=\mathbf{U}_{i} \mathbf{S}_{i} \mathbf{U}^{\mathrm{H}}
$$

where $\mathbf{S}_{i}=\operatorname{diag}\left[s_{1}\left(f_{i}\right), s_{2}\left(f_{i}\right), \ldots, s_{j}\left(f_{i}\right), \ldots, s_{n}\left(f_{i}\right)\right]$ is the diagonal matrix, it corresponds to the $i$ th discrete frequency $f_{i}$ containing a set of discrete singular values $s_{j}\left(f_{i}\right), \quad \mathbf{U}_{i}=$ $\left[\left\{u_{i 1}\right\},\left\{u_{i 2}\right\}, \ldots,\left\{u_{i j}\right\}, \ldots,\left\{u_{i n}\right\}\right]$ is the unitary matrix containing column singular vectors $\left\{u_{i j}\right\}$ it corresponds to the $i$-th discrete frequency $f_{i}, j=1,2, \ldots, n$, and $n$ denotes the dimension of $\mathbf{G}_{x x}\left(\mathrm{i} f_{i}\right)$ and is equal to number of measuring points in which the response of the structure was measured.

The decomposition given in Eq. (2) allows the modal parameter identification of a SDOF systems, i.e. the modal frequencies and mode shapes, at each singular value corresponding to the resonant frequency. Each set of the discrete singular values $s_{j}(f)$, for $j$ $=1,2, \ldots, n$, can be presented in the frequency domain as $n$ independent plots. The plot of the first singular values $s_{1}\left(f_{i}\right)$ is dominated by maximum values which, on the frequencyaxis, indicate a set of the modal frequencies of a structure $f_{k}$. According to the FDD theory, the first singular vectors $\left\{u_{i 1}\right\}$ of the unitary matrix $\mathbf{U}_{i}$ corresponding to the first maximum singular vales $s_{1}\left(f_{k}\right)$ are the $k$-th unscaled mode shape vectors corresponding to the $f_{k}$, thus

$$
\phi_{k}=\left\{u_{k 1}\right\} .
$$

The FDD procedure was adopted to write a computer program in Matlab, which was used for the numerical calculations. The PSDs and CSDs of the discrete digital signal, representing the free response of the considered footbridge, were estimated using the modified averaged periodogram method, called the Welch's technique. According to this technique the time series of the signal are split and then windowing procedure are overlapped before averaging them together. This technique minimizes the bias noise caused by the spectral leakage. As an example, the plots of the singular values $s_{1}(f)$ and $s_{2}(f)$ of the decomposed PSD matrices $\mathbf{G}_{x x}(\mathrm{i} f)$ of the free vibration responses of the footbridge girder "A" measured at the reference point and measurement point No. 23 are given in Fig. 4. Due to the field test results it was possible to identify first five specific vibration modes of the analysed footbridge. These normalised mode shapes and their theoretical approximation are shown in Fig. 6.

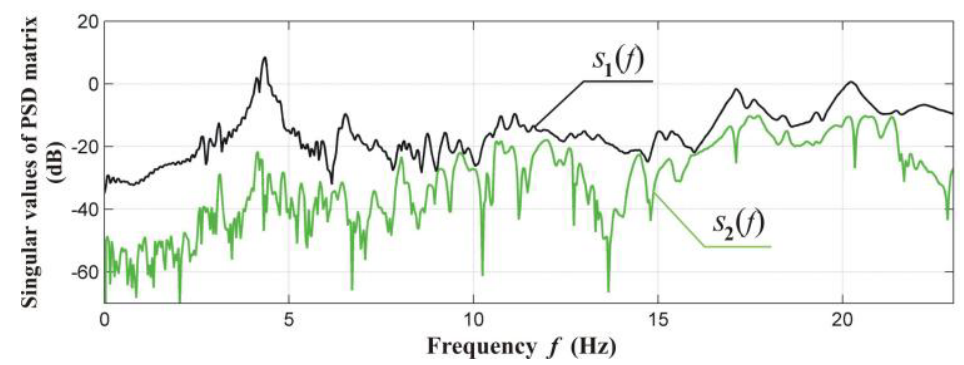

Fig. 4. Singular values of the decomposed PSD matrices of the free vibration responses of the footbridge girder "A" measured at the reference point and measuring point No. 23.

\subsection{Damping estimation}

The damping ratios of the footbridge were analysed on the basis of the free decays recorded only at the reference point. The analysed free vibration response was decomposed into five modal decays by isolating the contributions of the primary five of the most important modes of the footbridge. For this purpose, the filtering procedure using the 
eighth-order Type 1 Chebyshev band-pass digital filters with passband ripple $1 \mathrm{~dB}$ was applied. The following pass-bands in the filtering procedure were chosen: $3.5-5.0 \mathrm{~Hz}, 6.0$ 6.8 Hz, 11.0-11.5 Hz, 16.8-17.3 Hz, and 19.5-20.7 Hz. Fig. 5 (left side) depicts two modal decays provided by the filtering procedure with pass-band $3.5-5.0 \mathrm{~Hz}$ and $6.0-6.8 \mathrm{~Hz}$, regarding the natural frequencies $f_{1}=4.30 \mathrm{~Hz}$ and $f_{2}=6.59 \mathrm{~Hz}$, respectively.

Basing on the filtered data, containing only the contribution of a single mode, the modal damping ratios were estimated by a linear regression on the extremes of these modal decays (using the least square approximates). In this case, each $m$-th extreme value of the modal decay, denoted as $\hat{x}_{m}$, was found and the corresponding values $A_{m}$ were calculated according to the formula

$$
A_{m}=2 \cdot \ln \left(\frac{\hat{x}_{0}}{\left|\hat{x}_{m}\right|}\right)
$$

where $\hat{x}_{0}$ is the initial value of the modal decay.

Then, all $A_{m}$ values were plotted as separate points and were compared with a straight line obtained by a linear regression on $\Delta \cdot m$ regarding unknown logarithmic decrement $\Delta$. The damping ratio value was calculated according to the equation

$$
\xi=\frac{\Delta}{\sqrt{\Delta^{2}+4 \pi^{2}}} .
$$

Since the stability of the modal decays is limited, the use of the linear regression is helpful for assessment of the stability as well as for more precise estimation of the damping ratio. Fig. 5 (right side) depicts the linear regression on the extremes of two modal decays provided by the filtering procedure with pass-band $3.5-5.0 \mathrm{~Hz}$ and $6.0-6.8 \mathrm{~Hz}$. The obtained damping ratio values, corresponding to five mode shapes of the Fiberline Bridge presented in Fig. 6 , are $\xi_{1}=2.87 \%, \xi_{2}=1.63 \%, \xi_{3}=2.59 \%, \xi_{4}=2.09 \%$, and $\xi_{5}=2.57 \%$.
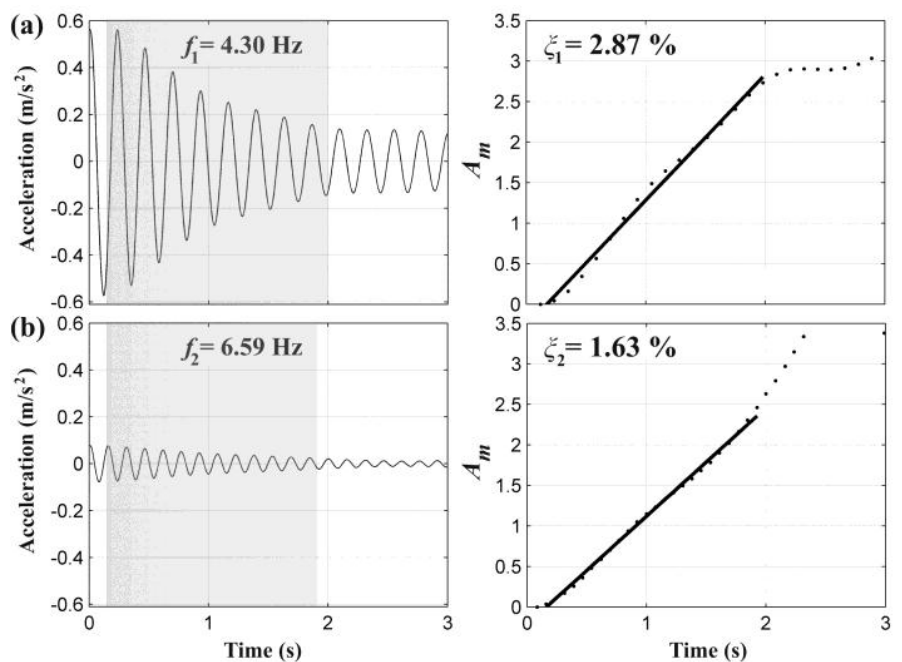

Fig. 5. Modal decays (left side) and linear regression on their extremes (right side) provided by filtering procedure using the eighth-order Type 1 Chebyshev band-pass digital filter with passband ripple $1 \mathrm{~dB}$ and pass-band (a) $3.5-5.0 \mathrm{~Hz}$ and (b) $6.0-6.8 \mathrm{~Hz}$. 


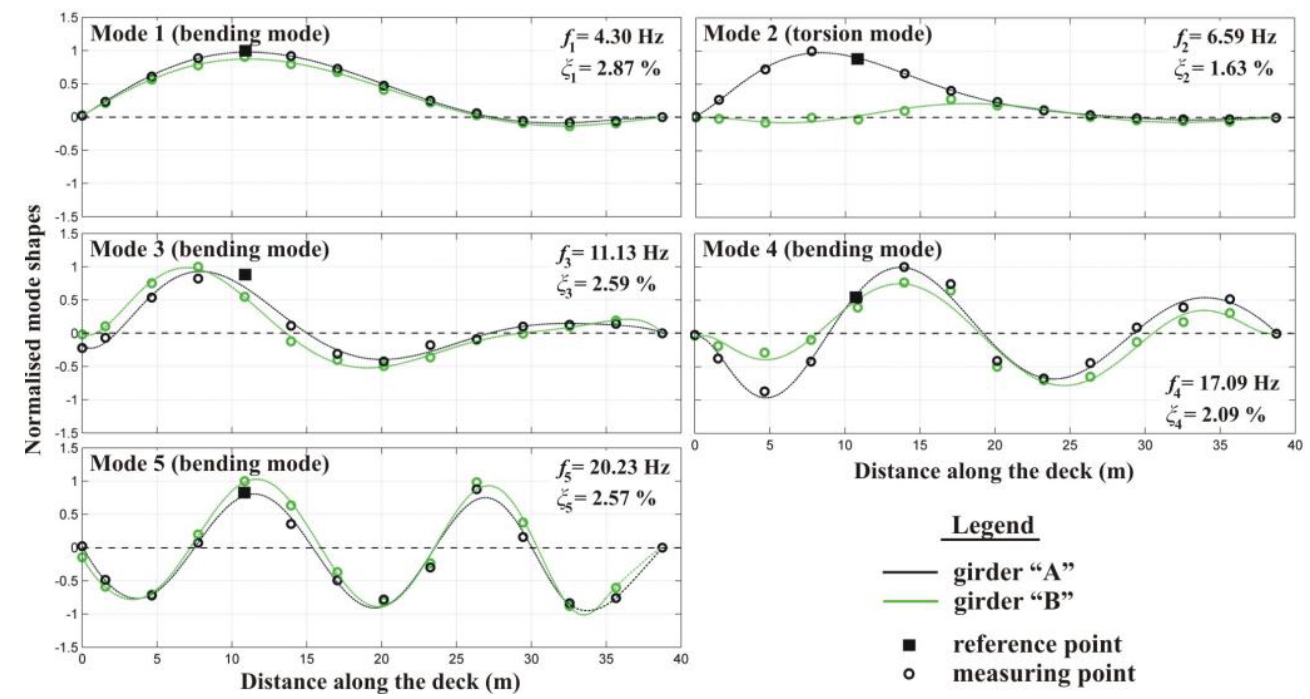

Fig. 6. Normalized mode shapes, corresponding to natural frequencies and damping ratios estimated from measured free vibration responses of the Fiberline Bridge.

\section{Comparison of the damping ratios of selected footbridges}

The comparison of the measured damping ratio values of the Fiberline Bridge with data published in the literature [14-16] for several selected footbridges made of various conventional materials without a control devices regarding their natural frequencies is presented in Fig. 7.

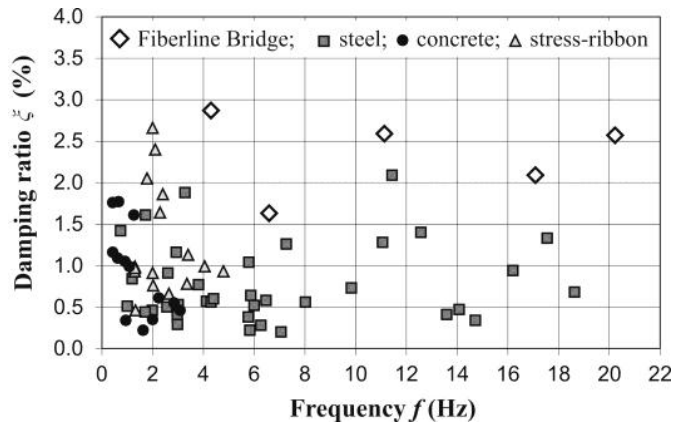

Fig. 7. Comparison of measured damping ratios of the Fiberline Bridge with data published in the literature for selected footbridges made of various materials regarding their natural frequencies.

\section{Conclusions}

The aim of this paper was to present methods and results of modal parameter identification of the all-GFRP composite cable-stayed Fiberline Bridge in Kolding, Denmark, based on in situ free-decay measurements using accelerometers. The application of an impulse due to human jumping in a single free vibration test was sufficient to excite the specific five vibration modes in the vertical direction of the analysed footbridge. In case of the used approaches the primary five natural frequencies, corresponding mode shapes and modal damping ratios were identified. The modes No. 1, 3, 4, and 5 are the vertical bending type of modes. The mode No. 2 is the untypical torsion mode of the footbridge deck as the result 
of vibrations mainly the girder "A", probably due to a relatively low bending-torsional stiffness of the deck system. In order to explain this problem the numerical analysis of free vibrations of the analysed footbridge will be made in the next stage.

The comparison of the estimated damping ratios of the Fiberline Bridge with data published in the literature for selected footbridges with comparable length made of various conventional materials showed that the modal damping of the cable-stayed footbridge made entirely of GFRP composite is relatively high. The values of the damping ratios of the analysed footbridge were within the range between $1.6 \%$ and $2.9 \%$ for all identified natural frequencies. The damping ratios for concrete footbridges were within the range of 0.2 to $1.8 \%$, for steel footbridges - within the range of 0.2 to $2.1 \%$, and for stress-ribbon footbridges - within the range of 0.5 to $2.7 \%$.

Nowadays, it is very advisable and valuable to conduct study in order to recognize the fundamental dynamic characteristics of all-GFRP footbridges. The collected information would be very useful for the procedure of the calculation model verification, the calculation model updating, the dynamic response prediction and the assessment of the technical state or structural health monitoring of this type of footbridges.

\section{References}

1. B. Stankiewicz, Procedia Engineer 40, 423 - 427 (2012)

2. A. Kabiri Ataabadi, S. Ziaei-Rad, H. Hosseini-Toudeshky, Steel Compos. Struct. 12, 53-72 (2011)

3. B. Stankiewicz, J. Compos. Mater. doi: 10.1177/0021998316669857 (to be published)

4. B. Stankiewicz, M. Tatara, J Civ. Eng. Architecture 9, 791-797 (2015)

5. A.B.S Santos Neto, H.L. La Rovere, Compos. Struct. 92, 2554-2564 (2010)

6. S. Robert, Bridging the World (Bridge Ink, Wilsonville, USA, 2003)

7. W.J. Harvey, Struct. Eng. Int. 4, 93 (1993)

8. M. Kutz, Handbook of materials selection (John Wiley \& Sons, 2002)

9. L. Yeou-Fong, B. Sainey, W.C. Walter, C. Yu-Tsung, Case Stud. Construction Mater. 1, 83-95 (2014)

10. Y. Bai, T. Keller, Compos. Struct. 82, 1 (2008)

11. R. Burgueńo, V.M. Karbhari, F. Seible, R.T. Kolozs, Compos. Struct. 54, 4 (2001)

12. Fiberline Composites Website, https:/fiberline.com/fiberline-bridge-kolding (accessed January 2017)

13. M. Batel, Sound Vib. 36, 8 (2002)

14. C. Butz, M. Feldmann, C. Heinemeyer, G. Sedlacek, B. Chabrolin, A. Lemaire, M. Lukic, P.O. Martin, E. Caetano, Á. Cunha, A. Goldack, A. Keil, M. Schlaich, Project RFS-CR-03019, Final Report (RFCS, Brussels, Belgium, 2007)

15. J.M.W. Brownjohn, M. Bocian, D. Hester, A. Quattrone, W. Hudson, D. Moore, S. Goh, M.S. Lim, J. Sound Vib. 384, 339-355 (2016)

16. K. Maes, K. Van Nimmen, E. Lourens, A. Rezayat, P. Guillaume, G. De Roeck, G. Lombaert, Mech. Syst. Signal Pr. 75, 245-260 (2016) 